DOI: 10.20472/IAC.2018.935.048

\author{
AVISHALOM TOR \\ Notre Dame Law School, United States
}

\title{
THE PSYCHOLOGY OF INEQUALITY IN ALLOCATION DECISIONS: THE EFFECT OF THE NUMBER OF RECIPIENTS (N)
}

\begin{abstract}
:
The present series of studies examines individuals' preferences for equality in the distribution of resources in the organization. In particular, we identify a ubiquitous factor in the organization that affects concerns about inequality-namely, the number of allocation recipients. More specifically, we tested the hypothesis that third-party decision makers who allocate resources become less concerned about inequality in the allocation as the number of recipients increases. We expected decision makers faced with a tradeoff between equality and other considerations, such as efficiency (in the sense of maximizing the overall value of the allocation) or equity (in the sense of rewarding recipients differently based on their inputs), to show a stronger preference for equality in allocations to a smaller number of recipients than in allocations to a larger number of recipients. Studies $1 \mathrm{a}$ and $1 b$ provide experimental evidence for the basic effect in a simple equality-efficiency tradeoff among participants in behavioral choice paradigms, while Study 2 provides further support for the effect in questionnaire responses concerning tradeoffs between equality and either efficiency or equity, and Study 3 further substantiates our findings using a large, representative cross-national sample of employed and unemployed individuals. Studies $4 a$ and $4 b$, respectively, examine possible mechanisms underlying the effect, showing, first, that social comparison-but not fairness considerations-appear to mediate the effect; and, second, that the effect holds even allocators know that recipients will not have information regarding others' allocations. Finally, Studies $5 \mathrm{a}$ and $5 \mathrm{~b}$ offer some manifestations of the effect in naturally-occurring data, in both faculty salary distributions at a large public university and in attitudes towards inequality in an extant, large-scale national survey.
\end{abstract}

\section{Keywords:}

Equality, distributive justice, efficiency, equity, social comparison 